\title{
Internet of Things versus Covid-19: Integrated Low- Cost Proposal for Oximetry Collection and Data Availability in Cloud for Strategic Management of Population in Isolation
}

\author{
Lucas Gonçalves Vilela Fernandez ${ }^{1}$, Rubens Tadeu Gonçalves ${ }^{2}$, Jean Carlos dos Santos \\ Moreira $^{3}$, Thaís Cordeiro Pereira ${ }^{4}$, Josué Felipe Pontes Nery ${ }^{5}$, Luiz Melk de Carvalho ${ }^{6}$, \\ Flávio Henrique Batista de Souza ${ }^{7 *}$
}

Centro Universitário de Belo Horizonte - UNIBH, Brazil

flabasouza@yahoo.com.br

\begin{abstract}
One of the challenges of countermeasures that governments around the world face to serve society is the monitoring of cases and the overcrowding of beds, in addition to the exposure of health professionals to the COVID-19. In a patient-focused analysis, monitoring that becomes necessary is oximetry. This research demonstrates an integrated solution for: collecting population oximetry data, concentrating collections in a cloud computing structure and generating epidemiological data, and a low-cost solution (several nations are in serious economic difficulties). The research had the following steps: to develop an oximetry device with a low cost market microprocessor, to integrate the oximeter developed with a web platform (to minimize the contact between healthy population, doctors and infected), to carry out real samples collections with the prototype and a recognized brand in the market (in order to compare the quality of the response obtained). There were 17 days of measurements, 3 times a day, in 3 patients, in Belo Horizonte city (3,000,000 inhabitants). An oximeter with the ESP32 microprocessor was developed due to its processing capacity. In the data collection, it forwards the measurements to a web structure, hosted on Cloud with a tab with a heat map of patient concentration. During the comparisons, the rates of: Beat per Minute (BPM) and SpO2 were verified. It was found $0 \%$ variation in oximetry measurements and a maximum variation of $7.25 \%$ BPM. The prototype is $60 \%$ cheaper than the competitor (in Brazilian Reais), adding population management capabilities in municipalities with low purchasing power.
\end{abstract}

Keywords: COVID-19, Oximetry, Cloud Computing, ESP32, Microprocessor 


\section{$3^{\text {rd }}$ International Conference on Research in SCIENCE, ENGINEERING AND TECHNOLOGY}

\section{Introduction}

The dissemination of CODIV-19 attacked 3 pillars of society: health, social life and financial conditions. One of the challenges of the countermeasures that governments face to serve society is the monitoring of cases and overcrowding of beds, in addition to the exposure of health professionals to the pandemic. In a patient-focused analysis, a monitoring parameter that becomes necessary is oximetry. The oxygen concentration in the blood is altered in the presence of COVID-19 in the patient (Coppo et al., 2020).

The current scenario of COVID-19 is chaotic, the lethality curve of the disease is only at the beginning of its journey and has already brought several deaths and financial losses. An exponentially complex curve, with many dependent variables, makes it very difficult to carry out data analysis and even to create adequate prevention models. Although the symptoms are similar to those of a common flu, the condition developed by the patient can worsen in a matter of one or two days, in this scenario the patient begins to present acute respiratory deficiency and needs adequate and well-trained medical care, in addition to a well-equipped location (Geier \& Geier, 2020).

Patients who have an aggravated disease need to be admitted to intensive care units, and may even need respiratory assistance through lung ventilators. There are some risk groups where the worsening of symptoms is seen more frequently. People who have some chronic diseases such as (putting diseases in the risk group) are much more susceptible to worsening (changing by synonym) of the condition, than people who have a stable and normal clinical picture, often being younger people.

Paying attention to the risk group, it will also show the vast majority of the elderly population. People in this public often already have chronic or acquired diseases during their lives and, aligning this with old age, end up becoming targets for disease, its worsening being practically inherent and, consequently, leading to its lethality. In view of its lethality and the contingency actions that are being taken all over the planet, it is necessary to raise and develop techniques, means and devices to more quickly identify the symptoms and be able to preserve the best possible health status, so that the treatment, in line with the immunity of the patient, can help them to overcome the virus (Rajgor et al., 2020; Spychalski et al. 2020).

Using the technique to read oximetry, together with the monitoring of other vital and physical signs, such as fatigue, it is possible to check if the individual is showing symptoms of the disease or if there is some worsening in the respiratory system. In many cases of COVID19 the patient, despite not realizing that they have the disease because it manifests in a silent way, presents hypoxia, that is, the decrease in oxygen rates in the tissues, making the body difficult to maintain its functions normal and extremely important for humans (Levitan, 2020).

In parallel to this scenario, technologies allow not only the collection of data, but their consolidation via the Internet, in order to keep the distance and make epidemiological data available, with low cost. These are technologies that were already on the rise, but the pandemic enabled their acceleration and enhancement, such as: cloud Computing, Internet of Things (IoT), Microprocessors (such as Arduino, ESP and others) and Sensors (Tuli et al., 2020; Çoban \& Çoban, 2020) 


\section{$3^{\text {rd }}$ International Conference on Research in SCIENCE, ENGINEERING AND TECHNOLOGY}

\section{Oxford - United Kingdom}

Thus, the main objective of this article is to demonstrate an integrated solution, capable of: collecting population oximetry data, concentrating collections in a cloud computing structure and generating epidemiological data (such as heat maps and case concentration). However, this objective is weighed by the low cost, since several nations are in serious economic difficulties due to the pandemic.

\section{Material and Methods}

\subsection{Concepts}

For the development of the research, some concepts were researched as references for the study, such as:

- Cloud Computing: According to Khan et al. (2020), the availability of services and resources over the internet can be considered as cloud computing, where the user consumes resources such as software, virtualized hardware and other services to implement their solutions.

- Internet of Things: According to Nauman et al. (2020), devices with lower processing capacities, considered "things" start, with the IoT, to have the ability to interconnect through computer networks and the internet, with the ability to transmit and receive data.

- REST (Representational State Transfer) or RESTFul architecture: it is based on micro services and uses the HTTP (Hypertext Transfer Protocol) protocols, which is the same basic protocol of the internet. In a REST micro service, there is a request made and a response returned. Usually the application makes the request passing the necessary values, the server processes and then returns the answer to the application. This response is usually in JSON (JavaScript Object Notation) format (Smith, 2020).

- Oximetry: is an exam used to measure the amount (in \%) of oxygen in the blood. This test is very important in the detection of pulmonary, cardiac and neurological diseases. The ideal level of oximetry in the blood must be greater than $90 \%$, but it can vary depending on the case of the patient or the situation in which the person is at the time (WHO, 2011). When this rate is below 90\% it indicates a deficiency of oxygenation in the blood and it is necessary to see the doctor to be corrected to the ideal levels. The patient may have hypoxemia, which is a serious condition, and causes severe tissue damage and depending on the level, there may be a risk of death. There are several methods to measure oximetry, one of these methods is from a device, called a "pulse oximeter", which is considered non-invasive. In the pulse oximeter the patient receives a device at the tip of their finger that emits internal lights and is able to capture it back. The red and infrared beams of light emitted allow the measurement of the oxygenation rate and other patient data such as heart rate and respiratory rate (Arias et al., 2020).

- OutSystems: a low-code platform that allows the creation of Web and mobile applications, uses very interactive user interfaces, has many development options bringing a variety of functionalities to the developed software (Jacinto et al., 2020). 


\section{$3^{\text {rd }}$ International Conference on Research in SCIENCE, ENGINEERING AND TECHNOLOGY}

- MAX30100: it is a sensor that has the capability to measure the oxygenation rate and heart rate. The sensor has two LEDs, a photo coupler and a processor optimized to perform the necessary analog-to-digital conversions. The chip works with a voltage range between $1.8 \mathrm{v}$ to $3.3 \mathrm{v}$. Its communication protocol follows the $\mathrm{I} 2 \mathrm{C}$ standard, which is a multi-master serial communication bus, originally created by Phillips and used to connect low speed peripherals to motherboards, microcontrollers and devices (Kalovrektis et al., 2020).

- ESP32: it is a System-On-Chip that manages to unite the main electronic components used in computing such as memory and processor, on a single chip, whether it is programmable or not. The chip has Wi-Fi and integrated Bluetooth, two processing cores, its own operating system to control its operation, as well as the reception of data through $\mathrm{Wi}-\mathrm{Fi}$ communication. One of the main characteristics of this module is the ability to use it with stand-alone operation, where only the card is necessary to provide wireless communication and also perform calculations with specific inputs and outputs. It can also provide this wireless communication medium to other cards, acting as an adapter module. The energy consumption of the module is low and there are also operating modes for better optimization of consumption, according to the needs of each prototype (Dokic et al., 2020).

- Arduino: is a low investment Open Source microcontroller, which makes it possible to create a huge range of devices that can interact with other people or with their own environments. It has inputs and outputs that can be used by the designer, according to their needs. In addition to the board itself, the Arduino can also be expanded using some additional boards, popularly called Shields. These cards add extra functionality to the Arduino, such as internet connection or Bluetooth connectivity (Dokic et al., 2020).

Such concepts guided the development of the prototype and the experiments carried out.

\subsection{Research Methodology}

The methodology of this work included the following steps:

- Development of an oximetry device with a low-cost market microprocessor and integration of the oximeter developed with a web platform (to minimize contact between healthy, medical and infected populations);

- carry out data collections with the prototype and a recognized equipment present on the market, in order to compare the quality of response obtained.

There were 17 days of measurements, 3 times a day, in 3 patients, in Belo Horizonte, a city of more than 3,000,000 inhabitants in Brazil.

\section{Results}

\subsection{Prototype Design}

In the initial prototype development phase, it was observed that the MAX30100 module that was acquired for the experiment, contained a design problem in the voltages used for the 


\section{$3^{\text {rd }}$ International Conference on Research in SCIENCE, ENGINEERING AND TECHNOLOGY}

communication of the $\mathrm{I} 2 \mathrm{C}$ protocol. The development with the Arduino platform was stable and after adjusting the MAX30100 module, the Arduino was able to receive the readings collected by the sensor. However, the Arduino does not have wireless connections natively, so it was necessary to use a separate chip or module, such as the ESP8266 chip or the ESP-01 module to provide this communication. For the continuity of the project, an evaluation of the processors used was performed, shown in Table 1.

Table 1: Microprocessors Evaluation

\begin{tabular}{|l|l|l|l|}
\hline & ESP32 & ESP8266 & Arduino UNO R3 \\
\hline Colours & 2 & 1 & 1 \\
\hline Architecture & 32 bits & $32 \mathrm{bits}$ & 8 bits \\
\hline Clock & $160 \mathrm{MHz}$ & $80 \mathrm{MHz}$ & $16 \mathrm{MHz}$ \\
\hline Wi-Fi & Yes & Yes & No \\
\hline Bluetooth & Yes & No & No \\
\hline RAM & $512 \mathrm{~KB}$ & $160 \mathrm{~KB}$ & $2 \mathrm{~KB}$ \\
\hline FLASH & $16 \mathrm{MB}$ & $16 \mathrm{MB}$ & $32 \mathrm{~KB}$ \\
\hline GPIO & 36 & 17 & 14 \\
\hline Interfaces & SPI/I2C/UART/I2S/CAN & SPI/I2C/UART/I2S & SPI/I2C/UART \\
\hline
\end{tabular}

Source: Authors, 2020.

Using the ESP-01 module, the Arduino was able to communicate wirelessly, via a standard Wi-Fi network in a satisfactory manner, taking around 3 to 5 seconds to communicate with the service created.

However, when combining the two functionalities, the reading of the signals along with the transmission over the WIFI network, a problem was shown to be recurrent. The sensor has an internal memory to store the readings with a limit of 16 addresses. The frequency of sensor reading, although not very high, is about $100 \mathrm{~Hz}$ and it ended up quickly filling the addresses available in the memory of the sensor, causing the so-called overflow, which is when the memory is full and the sensor tries to put more data in it.

Whenever this happened, the sensor turned off and it was necessary to restart it. This scenario was not ideal. Although it was possible to restart the sensor and continue with the readings, this was not viable in terms of stability, because every time it restarted it was necessary to go through the stabilization period again. In addition to being impracticable, there is also a risk of damaging the sensor and considerably shortening its useful life.

In order for the task to be carried out correctly, it was necessary to execute the tasks simultaneously because this way, the reading of the sensor could occur without interruptions and without having to wait for a return from the data sending function. With this procedure, it was possible to find a prototype board, called ESP32, where the big difference was, precisely, to have two colors and the ability to perform tasks in each of them, simultaneously. The communication with the sensor would take place using the same communication method used in Arduino, the I2C protocol, using an open source library, already consolidated.

After assembling the prototype, the structure of the service and its functionality, as well as the data model covering the proposed Web system, were created allowing the sensor to send data to this service. 


\section{$3^{\text {rd }}$ International Conference on Research in SCIENCE, ENGINEERING AND TECHNOLOGY}

After defining the model, the development of the Web part began by applying modern design patterns, such as the use of reactive components, application responsiveness, separation of layers. This allowed not only to have a robust application running with high quality and fluidity, but also to be used in devices with different screen sizes. To show the information in a more organized way, the concept of "cards" was used.

The architecture proposed for the study was that of micro services, with the objective of allowing technological independence, better maintainability and a capacity for integrating or processing data on other platforms.

Figure 1 shows the solution diagram. The sensors collect the oximetry measurement. With this architectural pattern, the prototype sends the data collected through the services, and they are responsible for saving and treating this data in the manner previously established, in this case the REST architecture.

After collections are processed by the REST service, they are stored in a database, hosted in the cloud. This database presents its results in a data analytics structure developed and hosted in the cloud, for the access of allowed users.

Figures 2 and 3 demonstrate the web interfaces for evaluations in data Analytics, with readings indications, heat maps that can demonstrate where the measurements were made (in the case of Belo Horizonte city) and, mainly, readings and locations of patients who presented oximetry characteristics that can demonstrate a progression of COVID-19.

Figure 4 shows the individual assessment of the patient (WEB system). In this case, both the patient and the responsible doctor or nurse can monitor the oximetry analysis.

Figure 1: Prototype Diagram.

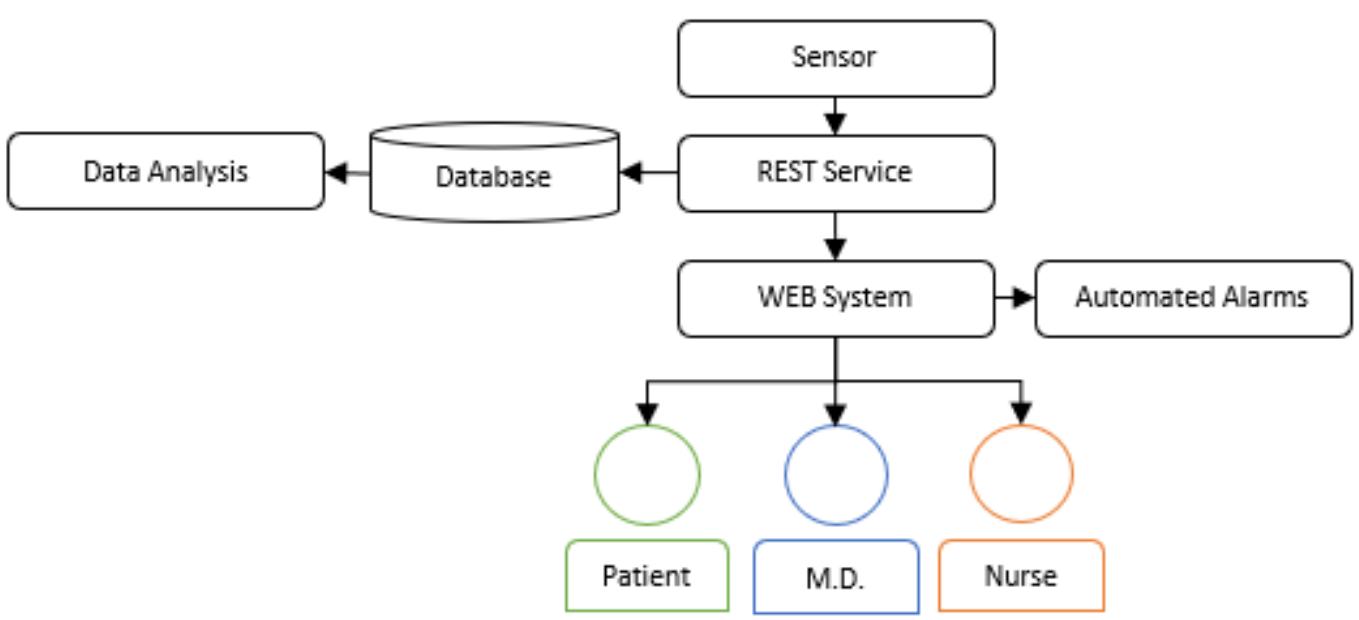

Source: Authors, 2020 


\section{$3^{\text {rd }}$ International Conference on Research in SCIENCE, ENGINEERING AND TECHNOLOGY}

11-13 March, 2021

Oxford - United Kingdom

Figure 2: Dashboard allocated on the web, in a structure based on cloud computing, to demonstrate the consolidation of data collected from oximetry (in Portuguese - viable translation).

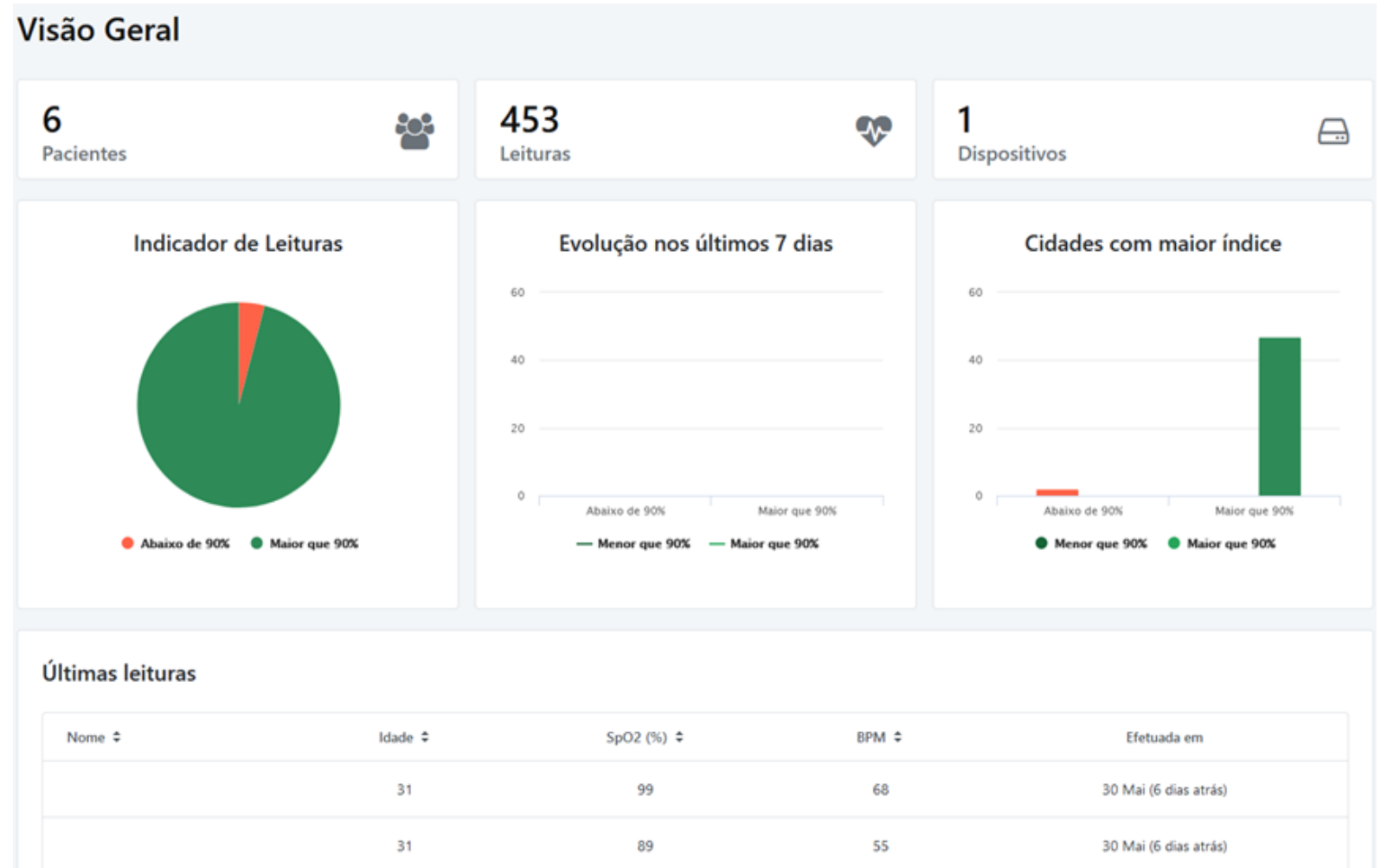

Translating: 6 Patients; 453 records; 1 device; Records Indicator; Evolution in 7 days; Highest Index Cities; Last Records.

Source: Authors, 2020. 


\section{$3^{\text {rd }}$ International Conference on Research in SCIENCE, ENGINEERING AND TECHNOLOGY}

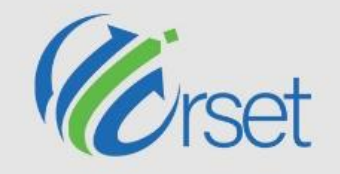

11-13 March, 2021

Oxford - United Kingdom

Figure 3: Heat maps of concentration of monitored patients (Belo Horizonte city)

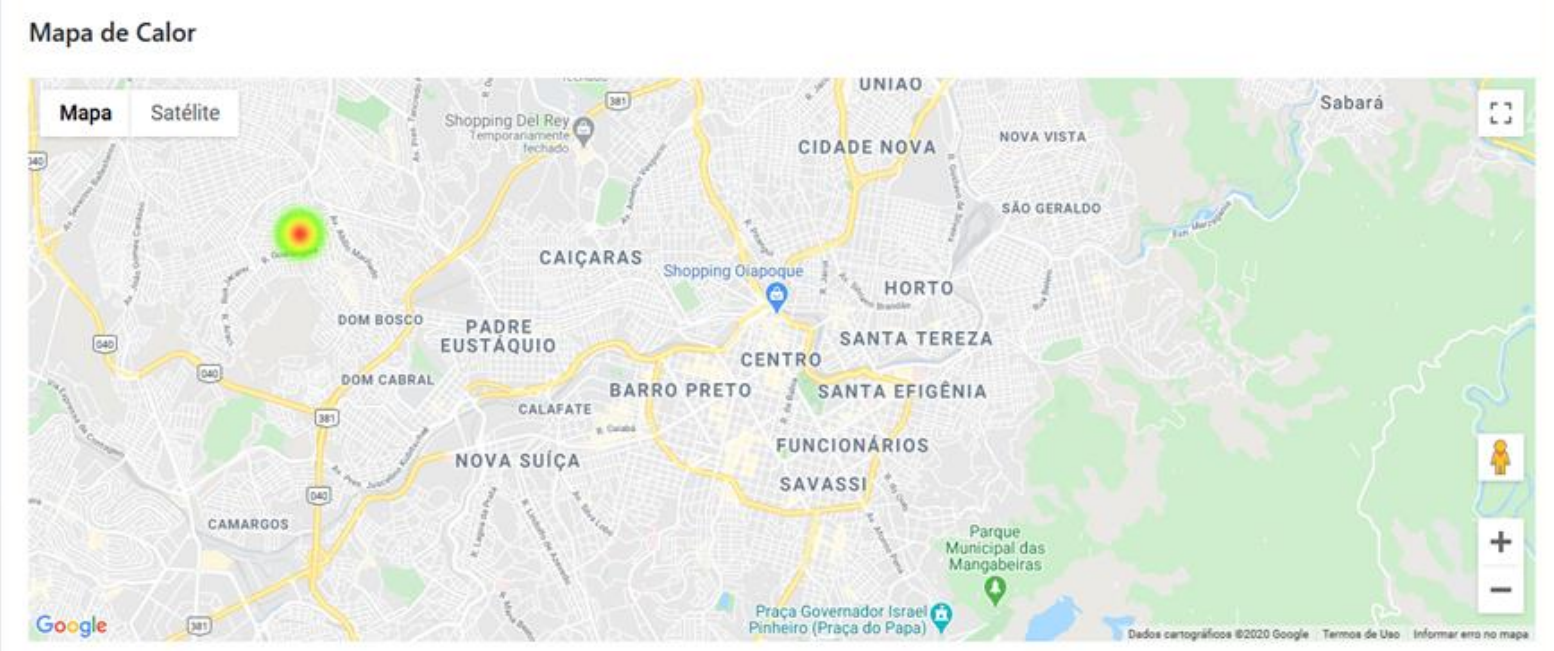

Source: Authors, 2020.

Figure 4: Patient Individual Evaluation

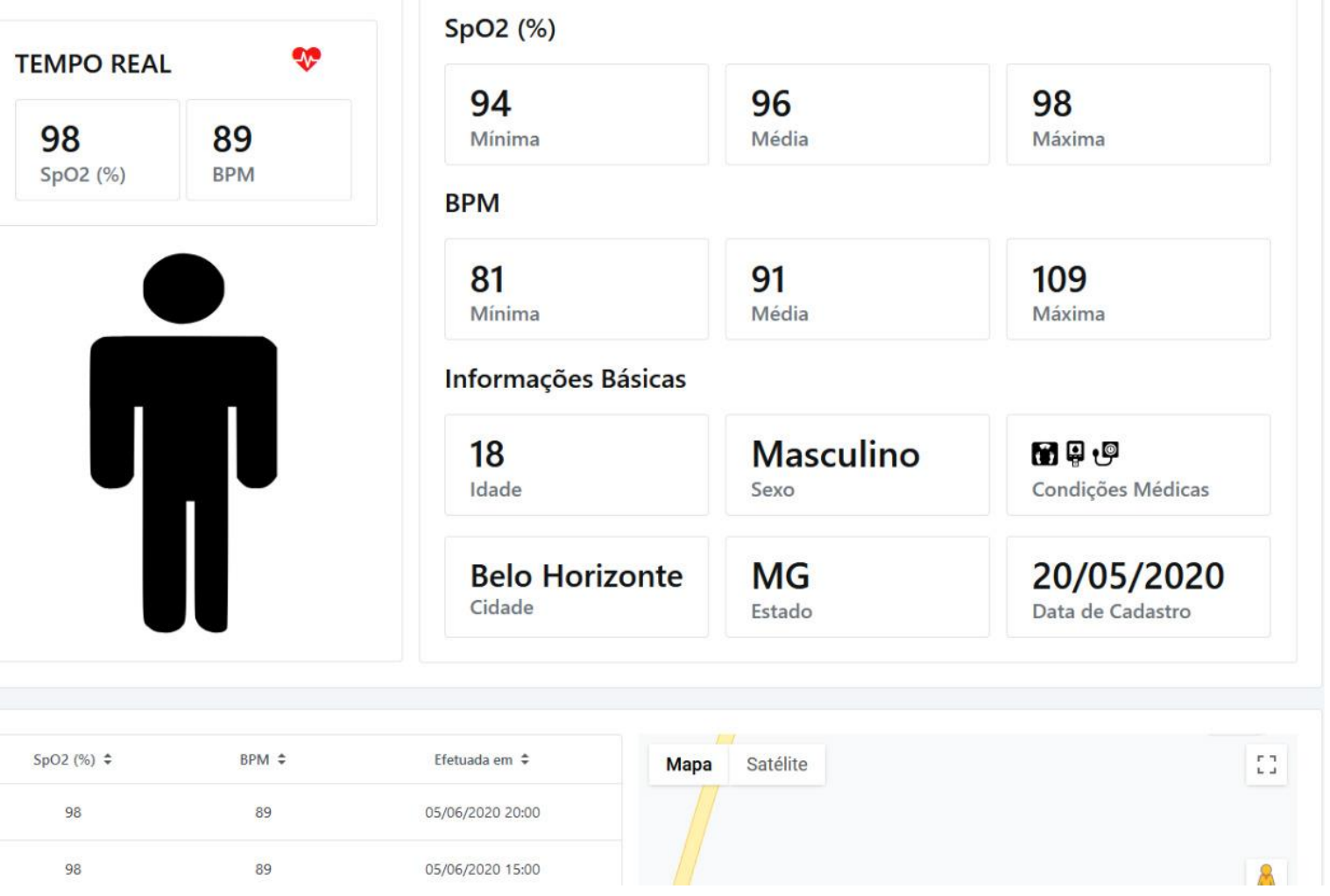

Source: Authors, 2020. 


\section{$3^{\text {rd }}$ International Conference on Research in SCIENCE, ENGINEERING AND TECHNOLOGY}

11-13 March, 2021

Oxford - United Kingdom

\subsection{Experiments and Comparisons}

With the system in full operation, the stage of validation and tests were initiated, in order to assess what would be the reading quality delivered by the sensor and its effectiveness for the purpose. The test performed was a comparison, using a commercially acquired and calibrated oximeter. With the competitor and the prototype side by side, it is expected its stabilization stage that lasts about 5 to 10 seconds and, after that, the measured values are recorded for later evaluation and comparison.

The samples were collected in 17 days, during the three periods of the day, with a collection performed in the morning, one in the afternoon and the last at night. Three people volunteered for the experiment. A comparative graphical evaluation is demonstrated in Appendix I

The competing oximeter has a calibration report carried out in 2018.

During the comparisons, the rates of Beat per Minute (BPM) and $\mathrm{SpO} 2$, it was found $0 \%$ variation in oximetry measurements and a maximum variation of $7.25 \%$ BPM. Finally, the prototype proved to be $60 \%$ cheaper than the competitor (analysis in Brazilian Reais), adding population management capabilities in municipalities with low purchasing power.

\section{Conclusion}

The general objective of this work was to produce a low-cost oximeter for better health monitoring, and which would also serve as an aid during periods of COVID-19.

The developed solution proved to be efficient in reading questions, capable of demonstrating information of: number of patients, number of readings, measurement date and time, monitoring by device, evolution in the last 7 days, cities with the highest index (for state or national analyzes), patient age, oximetry, BPM and patient concentration heat maps. The solution proves to be affordable in economic terms.

In addition, one can also mention the benefits of a greater generation of statistical data, where they may be processed in the future using other tools or technologies in order to obtain specific relationships and comparisons, and the current understanding of different clinical conditions can be improved, symptoms or diseases. In an increasingly globalized world, data becomes increasingly necessary and is extremely important in helping to make simple or even complex decisions, as is constantly noted in the health area.

\section{References}

[1] Coppo, A., Bellani, G., Winterton, D., Di Pierro, M., Soria, A., Faverio, P., Cairo, M., Mori, S., Messinesi, G., Contro, E., Bonfanti, P., Benini, A., Valsecchi, M. G., Antolini, L., Foti, G. (2020). Feasibility and physiological effects of prone positioning in non-intubated patients with acute respiratory failure due to COVID-19 (PRON-COVID): a prospective cohort study. The Lancet Respiratory Medicine, 8(8), 765-774.

[2] Geier, M. R., \& Geier, D. A. (2020). Respiratory conditions in coronavirus disease 2019 (COVID-19): Important considerations regarding novel treatment strategies to reduce mortality. Medical Hypotheses, 109760. 


\section{$3^{\text {rd }}$ International Conference on Research in SCIENCE, ENGINEERING AND TECHNOLOGY}

11-13 March, 2021

Oxford - United Kingdom

[3] Rajgor, D. D., Lee, M. H., Archuleta, S., Bagdasarian, N., \& Quek, S. C. (2020). The many estimates of the COVID-19 case fatality rate. The Lancet Infectious Diseases, 20(7), 776777.

[4] Spychalski, P., Błażyńska-Spychalska, A., and Kobiela, J. (2020). Estimating case fatality rates of COVID-19. The Lancet. Infectious Diseases.

[5] Levitan, R. M. (2020). Pulse Oximetry as a Biomarker for Early Identification and Hospitalization of COVID- 19 Pneumonia. Academic Emergency Medicine, 27(8), 785786.

[6] Tuli, S., Tuli, S., Tuli, R., and Gill, S. S. (2020). Predicting the Growth and Trend of COVID-19 Pandemic using Machine Learning and Cloud Computing. Internet of Things, 100222.

[7] Çoban, A., and Çoban, N. (2020). Determining of the spring constant using Arduino. Physics Education, 55(6), 065028.

[8] Khan, M. K., Shuja, J., Jararweh, Y., Yu, G., Guizani, M., Verikoukis, C. and Ahmad, R. W. (2020). IEEE Access Special Section Editorial: Mobile Edge Computing and Mobile Cloud Computing: Addressing Heterogeneity and Energy Issues of Compute and Network Resources. IEEE Access, 8, 163769-163774.

[9] Nauman, A., Qadri, Y. A., Amjad, M., Zikria, Y. B., Afzal, M. K., and Kim, S. W. (2020). Multimedia Internet of Things: A comprehensive survey. IEEE Access, 8, 8202-8250.

[10] Smith, B. (2020). JSON básico: conheça o formato de dados preferido da web. Novatec Editora.

[11] WHO. Global Pulse Oximetry Project. 2011. Disponível em:<https://www.who.int/patientsafety/safesurgery/pulse_oximetry/en/>. Acessado em: 20.04.2020.

[12] Arias, A. C., Lochner, C., Pierre, A., and Khan, Y. (2020). U.S. Patent No. 10,548,519. Washington, DC: U.S. Patent and Trademark Office.

[13] Jacinto, A., Lourenço, M., and Ferreira, C. (2020, October). Test mocks for low-code applications built with OutSystems. In Proceedings of the 23rd ACM/IEEE International Conference on Model Driven Engineering Languages and Systems: Companion Proceedings (pp. 1-5).

[14] Kalovrektis, K., Korinthios, I., Xenakis, A., Gotsinas, A., and Stamoulis, G. 802.15. 4based Efficient Wireless Sensor System Design for Monitoring Blood Oxygen and Heart Rate in IoT Medical Applications. Journal of Wireless Networking and Communications 2020, 10(1): 9-16

[15] Dokic, K., Radisic, B., and Cobovic, M. (2020, March). MicroPython or Arduino C for ESP32-Efficiency for Neural Network Edge Devices. In International Symposium on Intelligent Computing Systems (pp. 33-43). Springer, Cham. 
$3^{\text {rd }}$ International Conference on Research in SCIENCE, ENGINEERING AND TECHNOLOGY

\section{Cirset}

11-13 March, 2021

Oxford - United Kingdom

Appendix I - Comparisons of Oximetry and BPM measurements of the prototype against another market meter (Example: Patient 1)

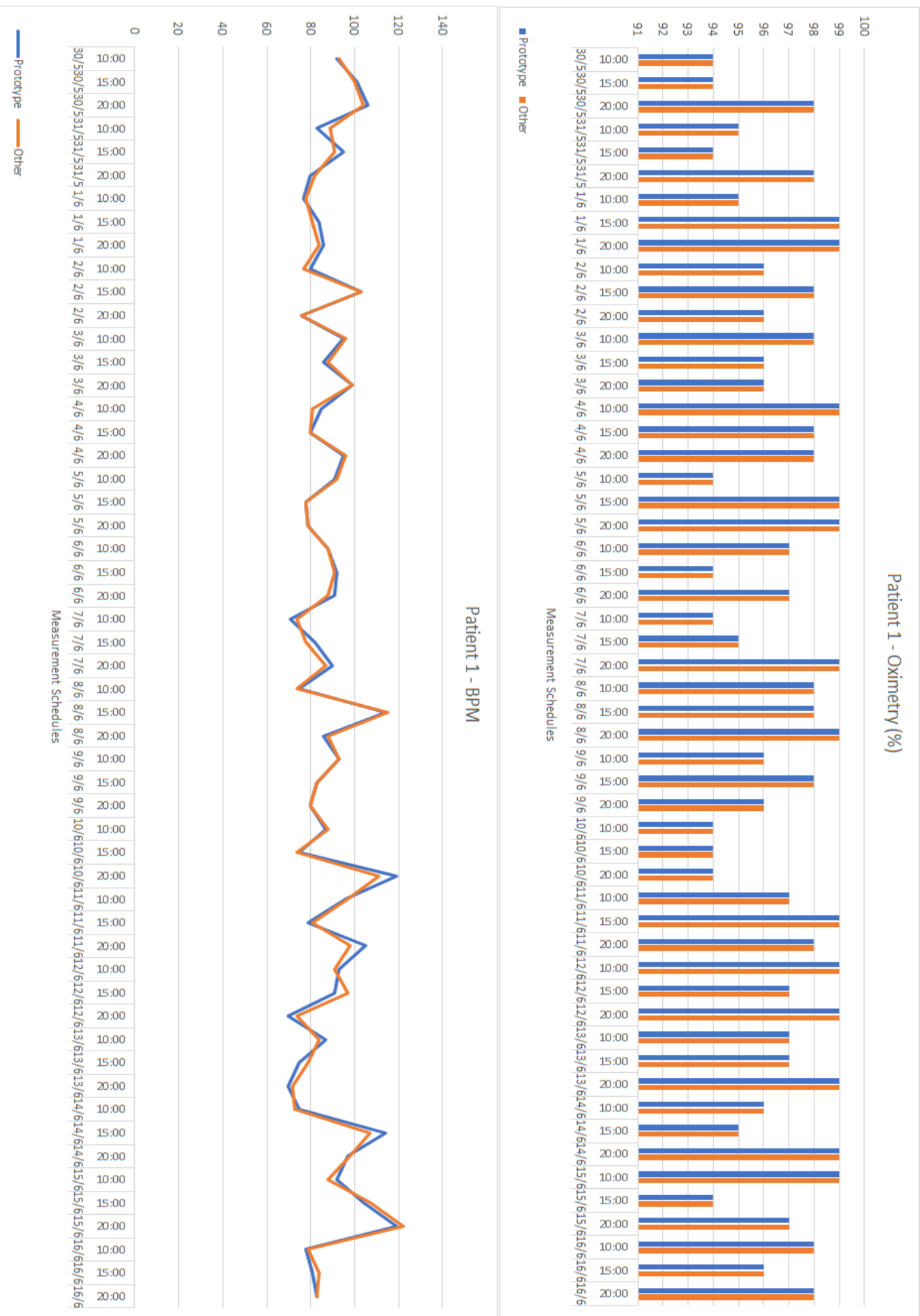

